\title{
ON THE BOUNDARY OF MODULI SPACES OF LOG HODGE STRUCTURES, II: NONTRIVIAL TORSORS
}

\author{
TATSUKI HAYAMA
}

\begin{abstract}
In this paper, we determine when a natural torsor arising in the work of Kato and Usui on partial compactification of period domains of pure Hodge structure is trivial, and we give an application to cycle spaces.
\end{abstract}

\section{$\S 1$. Introduction}

Let $D$ be a period domain of pure Hodge structures defined by Griffiths [G]. A variation of $\mathbb{Z}$-Hodge structure over the $n$-product of punctured disk $\left(\Delta^{*}\right)^{n}$ gives the period map $\left(\Delta^{*}\right)^{n} \rightarrow \Gamma \backslash D$, where $\Gamma$ is the monodromy group, that is, the $\mathbb{Z}$-module generated by the monodromy transformations. We assume that the monodromy transformations are unipotent. In this article, we treat partial compactifications of $\Gamma \backslash D$ so that the period map is extended over $\Delta^{n}$.

In the case where $D$ is Hermitian symmetric, Ash, Mumford, Rapoport, and Tai in [AMRT] gave partial compactifications of $\Gamma \backslash D$ (and also compactifications of arithmetic quotients of $D$ ). Later, Kato and Usui in [KU] generalized toroidal partial compactifications for any period domain $D$ which is not Hermitian symmetric in general, and showed that these are moduli spaces of $\log$ Hodge structures. This partial compactification is given by using toroidal embedding associated to the cone generated by the data of the monodromy. In fact, for generators $T_{1}, \ldots, T_{n}$ of the monodromy group $\Gamma$, the partial compactification $\Gamma \backslash D_{\sigma}$ is given by the cone $\sigma=\sum_{j=1}^{n} \mathbb{R}_{\geq 0} N_{j}$ $\left(N_{j}=\log T_{j}\right)$ in the Lie algebra. Here a boundary point is a nilpotent orbit associated to a face of $\sigma$ (see Section 3.1).

In the classical situation (i.e., $D$ is Hermitian symmetric), $\Gamma \backslash D_{\sigma}$ is an analytic space. In contrast, for general period domains, $\Gamma \backslash D_{\sigma}$ may not be

Received October 17, 2011. Revised December 11, 2012. Accepted January 19, 2013.

First published online November 19, 2013.

2010 Mathematics Subject Classification. Primary 32G20.

The author was supported by the National Science Council of Taiwan.

(C) 2014 by The Editorial Board of the Nagoya Mathematical Journal 
an analytic space. In fact, the boundary components of the partial compactification $\Gamma \backslash D_{\sigma}$ can have codimension greater than 1 , although it is 1 in the classical situation (see Example 3.3). This means that there can be slits on the boundary of $\Gamma \backslash D_{\sigma}$. Kato and Usui [KU] define logarithmic manifolds as a generalization of analytic spaces and state that $\Gamma \backslash D_{\sigma}$ is a logarithmic manifold.

A part of the geometric structure of $\Gamma \backslash D_{\sigma}$ is given by a torsor $E_{\sigma} \rightarrow \Gamma \backslash D_{\sigma}$ constructed in [KU] (see Section 3.1). We will discuss these torsors. Our main result is the following.

TheOREM 1.1 (see Theorem 3.4). The torsor $E_{\sigma} \rightarrow \Gamma \backslash D_{\sigma}$ is trivial if and only if $D$ is Hermitian symmetric or $\sigma=\{0\}$.

In [H1], we proved this result in the case where $D$ is Hermitian symmetric. In this paper, we treat the case where $D$ is not Hermitian symmetric. To prove Theorem 1.1, we use a result from Fels, Huckleberry, and Wolf [FHW, Theorem 4.4.3] to show that, unless D is Hermitian symmetric, any holomorphic function on $D$ is constant.

We used a different strategy to prove the nontriviality of the torsors for the one example in [H1, Proposition 5.8]. Generalizing this approach, we give another proof of the nontriviality result in Proposition 4.3. This second proof is stronger than the first one since it gives a nontriviality on some open sets around a boundary point. We use cycle spaces and the SL(2)-orbit theorem there. Some property of cycle spaces induces the nontriviality.

In conclusion, the properties of cycle spaces induce the above nontriviality results. Cycle spaces can have significance in the study of moduli spaces of log Hodge structures in a wider framework. In fact, the property of cycle spaces of Lemma 4.1 induces our later work [H2]. On the other hand, Green, Griffiths, and Kerr [GGK2] have introduced Mumford-Tate domains as a generalization of period domains, and they also indicate the importance of cycle space in relation to cohomology groups of Mumford-Tate domains. Moreover, Kerr and Pearlstein $[\mathrm{KP}]$ have constructed partial compactifications of Mumford-Tate domains in the same manner as Kato and Usui $[\mathrm{KU}]$. We expect that our results will fit into the case for the boundaries of the Mumford-Tate domains.

This article has the following organization. In Section 2.1, we review period domains of Hodge structures. In Sections 2.2 and 2.3, we discuss cycle spaces of period domains. In Section 3, we review moduli spaces of 
polarized log Hodge structures. In Section 4, we reformulate our prior results from [H1] in terms of cycle spaces.

\section{$\S 2$. Cycle spaces of period domains}

\subsection{Polarized Hodge structures and period domains}

We recall the definition of polarized Hodge structures and of period domains. A Hodge structure of weight $w$ with Hodge numbers $\left(h^{p, q}\right)_{p, q}$ is a pair $\left(H_{\mathbb{Z}}, F\right)$ consisting of a free $\mathbb{Z}$-module of rank $\sum_{p, q} h^{p, q}$ and a decreasing filtration on $H_{\mathbb{C}}:=H_{\mathbb{Z}} \otimes \mathbb{C}$ satisfying the following conditions:

(H1) $\operatorname{dim}_{\mathbb{C}} F^{p}=\sum_{r \geq p} h^{r, w-r}$ for all $p$;

(H2) $H_{\mathbb{C}}=\bigoplus_{p+q=w} H^{p, q}\left(H^{p, q}:=F^{p} \cap \overline{F^{w-p}}\right)$.

A polarization $\langle$,$\rangle for a Hodge structure \left(H_{\mathbb{Z}}, F\right)$ of weight $w$ is a nondegenerate bilinear form on $H_{\mathbb{Q}}:=H \otimes \mathbb{Q}$, symmetric if $w$ is even and skewsymmetric if $w$ is odd, satisfying the following conditions:

(P1) $\left\langle F^{p}, F^{q}\right\rangle=0$ for $p+q>w$;

(P2) $i^{p-q}\langle v, \bar{v}\rangle>0$ for $0 \neq v \in H^{p, q}$.

We fix a polarized Hodge structure $\left(H_{\mathbb{Z}, 0}, F_{0},\langle,\rangle_{0}\right)$ of weight $w$ with Hodge numbers $\left(h^{p, q}\right)_{p, q}$. We define the set of all Hodge structures of this type

$$
D:=\left\{\begin{array}{l|l}
F & \begin{array}{c}
\left(H_{\mathbb{Z}, 0}, F,\langle,\rangle_{0}\right) \text { is a polarized Hodge structure } \\
\text { of weight } w \text { with Hodge numbers }\left(h^{p, q}\right)_{p, q}
\end{array}
\end{array}\right\} .
$$

The space $D$ is called the period domain. Moreover, we have the flag manifold

$$
\check{D}:=\left\{\begin{array}{l|l}
F & \begin{array}{c}
\left(H_{\mathbb{Z}, 0}, F,\langle,\rangle_{0}\right) \text { satisfies the conditions } \\
(\mathrm{H} 1),(\mathrm{H} 2), \text { and }(\mathrm{P} 1)
\end{array}
\end{array}\right\} .
$$

The space $\check{D}$ is called the compact dual of $D$, and it contains $D$ as an open subset. Let $G_{A}:=\operatorname{Aut}\left(H_{A, 0},\langle,\rangle_{0}\right)$. Then, $G_{\mathbb{R}}$ acts transitively on $D$, and $G_{\mathbb{C}}$ acts transitively on $\check{D}$. The group $G_{\mathbb{R}}$ is a classical group such that

$$
G_{\mathbb{R}} \cong \begin{cases}\operatorname{Sp}(h, \mathbb{R}) & \text { if } w \text { is odd } \\ \operatorname{SO}\left(h_{\text {odd }}, h_{\text {even }}\right) & \text { if } w \text { is even }\end{cases}
$$

where $2 h=\operatorname{rank} H_{\mathbb{Z}}, \operatorname{Sp}(h, \mathbb{R})$ is the $(2 h \times 2 h)$-matrix symplectic group, $h_{\text {odd }}=\sum_{p \text { : odd }} h^{p, q}$, and $h_{\text {even }}=\sum_{p: \text { even }} h^{p, q}$. 
Let $\mathfrak{g}_{A}=\operatorname{Lie} G_{A}(A=\mathbb{R}, \mathbb{C})$. We then have the decomposition $\mathfrak{g}_{\mathbb{C}}=$ $\bigoplus_{p+q=0} \mathfrak{g}^{p, q}$ given by

$$
\mathfrak{g}^{p, q}=\left\{\alpha \in \mathfrak{g}_{\mathbb{C}} \mid \alpha H^{p^{\prime}, q^{\prime}} \subset H^{p+p^{\prime}, q+q^{\prime}} \text { for } p^{\prime}, q^{\prime} \in \mathbb{Z}\right\}
$$

with respect to a Hodge decomposition $H_{\mathbb{C}}=\bigoplus H^{p, q}$.

EXAMPLE 2.1 (Upper half-plane). Let us consider the case where the Hodge numbers $h^{1,0}=h^{0,1}=1,0$ otherwise. Then the corresponding classifying space $D$ is the upper half-plane $\{z \in \mathbb{C} \mid \operatorname{Im} z>0\}$, and $\check{D} \cong \mathbb{P}^{1}$. We have $G_{A} \cong \operatorname{SL}(2, A)(A=\mathbb{Z}, \mathbb{R}, \mathbb{C})$, where the action of $\operatorname{SL}(2, \mathbb{C})$ on $\check{D}$ is given by the linear fractional transformation. Here $\mathfrak{g}_{\mathbb{R}}=\mathfrak{s l}(2, \mathbb{R})$ is generated by

$$
\mathbf{n}_{-}=\left(\begin{array}{cc}
0 & 1 \\
0 & 0
\end{array}\right), \quad \mathbf{h}=\left(\begin{array}{cc}
-1 & 0 \\
0 & 1
\end{array}\right), \quad \mathbf{n}_{+}=\left(\begin{array}{ll}
0 & 0 \\
1 & 0
\end{array}\right)
$$

We call the triple the $s l_{2}$-triple. The $s l_{2}$-triple satisfies the following conditions:

$$
\left[\mathbf{n}_{+}, \mathbf{n}_{-}\right]=\mathbf{h}, \quad\left[\mathbf{n}_{ \pm}, \mathbf{h}\right]= \pm 2 \mathbf{n}_{ \pm}
$$

The Hodge decomposition of $\mathfrak{g}_{\mathbb{C}}$ with respect to $i \in D$ is given by

$$
\mathfrak{g}^{-1,1}=\mathbb{C}\left(i \mathbf{n}_{-}-\mathbf{h}+i \mathbf{n}_{+}\right), \quad \mathfrak{g}^{0,0}=\mathbb{C}\left(\mathbf{n}_{-}-\mathbf{n}_{+}\right), \quad \mathfrak{g}^{1,-1}=\overline{\mathfrak{g}^{-1,1}}
$$

Returning to the general case, the isotropy subgroup $L$ of $G_{\mathbb{R}}$ at $F_{0}$ is given by

$$
\begin{aligned}
L & =\left\{g \in G_{\mathbb{R}} \mid g F_{0}=F_{0}\right\} \\
& \cong \begin{cases}\prod_{p \leq m} U\left(h^{p, q}\right) & \text { if } w=2 m+1, \\
\prod_{p<m} U\left(h^{p, q}\right) \times \operatorname{SO}\left(h^{m, m}\right) & \text { if } w=2 m .\end{cases}
\end{aligned}
$$

They are compact subgroups of $G_{\mathbb{R}}$ but not maximal compact unless $D$ is Hermitian symmetric. We define

$$
H^{\text {even }}=\bigoplus_{p: \text { even }} H_{0}^{p, q}, \quad H^{\text {odd }}=\bigoplus_{p: \text { odd }} H_{0}^{p, q}
$$


where $H_{\mathbb{C}}=\bigoplus H_{0}^{p, q}$ is the Hodge decomposition for $F_{0}$. Here

$$
\begin{aligned}
K & =\left\{g \in G_{\mathbb{R}} \mid g H^{\text {even }}=H^{\text {even }}\right\} \\
& \cong \begin{cases}U(h) & \text { if } w \text { is odd }, \\
S\left(O\left(h_{\text {odd }}\right) \times O\left(h_{\text {even }}\right)\right) & \text { if } w \text { is even }\end{cases}
\end{aligned}
$$

is the maximal subgroup containing $L$ (see [CMP, Example 4.3.6], [LS, Lemma 2.8]). By the connectivity of $G_{\mathbb{R}}, D$ is connected if $w$ is odd, $D$ has two connected components if $w$ is even, and $h_{\text {even }}, h_{\text {odd }}>0$. Here $D$ is Hermitian symmetric if and only if the isotropy subgroup is a maximally compact subgroup, that is, if one of the following is satisfied (see [U1, (1.8)]):

(1) $w=2 m+1, h^{p, q}=0$ unless $p=m+1, m$;

(2) $w=2 m, h^{p, q}=1$ for $p=m+1, m-1, h^{m, m}$ is arbitrary, $h^{p, q}=0$ otherwise;

(3) $w=2 m, h^{p, q}=1$ for $p=m+a, m+a-1, m-a, m-a+1$ for some $a \geq$ $2, h^{p, q}=0$ otherwise.

In the case (1), $D$ is a Hermitian symmetric domain of type III. In the case (2) or (3), an irreducible component of $D$ is a Hermitian symmetric domain of type IV. We call the cases (1)-(3) the classical situation.

EXAMPLE 2.2 (The weight 1 case). We give an example of period domains of weight 1 and $h^{1,0}=h^{0,1}=n, 0$ otherwise. This case corresponds to case (1) above. Now $G_{A}=\operatorname{Sp}(n, A)(A=\mathbb{Z}, \mathbb{R}, \mathbb{C})$ and

$$
\begin{aligned}
D & =\{W:\langle,\rangle \text {-isotropic } n \text {-planes } \mid W>0 \text { for } i\langle\bullet, \bar{\bullet}\rangle\} \\
& \cong\left\{Z \in \mathbb{C}^{n \times n} \mid I-Z Z^{*}>0\right\} \\
& \cong \operatorname{Sp}(n, \mathbb{R}) / U(n),
\end{aligned}
$$

where $>0$ means positive definite. The space $D$ is called the Siegel space of degree $n$ (see $[\mathrm{N}]$ for details).

\subsection{Cycle spaces of period domains}

Let $D_{0}$ be an irreducible component including $F_{0}$ of a period domain $D$. Then the identity component $G_{\mathbb{R}, 0}$ acts on $D_{0}$ transitively. Let $K_{0}$ be the maximal compact subgroup of $G_{\mathbb{R}, 0}$ containing the isotropy subgroup $L_{0}$ at $F_{0}$. We then have the real analytic projection

$$
p: D_{0} \cong G_{\mathbb{R}, 0} / L_{0} \rightarrow G_{\mathbb{R}, 0} / K_{0}
$$


If $w$ is odd,

$$
G_{\mathbb{R}, 0} / K_{0}=\operatorname{Sp}(h, \mathbb{R}) / U(h)
$$

is the Hermitian symmetric domain of Example 2.2. If $w$ is even,

$$
G_{\mathbb{R}, 0} / K_{0}=\mathrm{SO}_{0}\left(h_{\text {odd }}, h_{\text {even }}\right) / \mathrm{SO}\left(h_{\text {odd }}\right) \times \mathrm{SO}\left(h_{\text {even }}\right)
$$

is a symmetric space which does not have any complex structure unless the projection $p$ is trivial (see [CMP, Example 4.3.6]). Moreover, $G_{\mathbb{R}, 0} / K_{0}$ is written as the set of all $H_{\text {even }}$ for $F \in D_{0}$ (see [LS, Lemma 2.10]), and the projection $p$ is given by

$$
F \mapsto H_{\text {even }}
$$

Then it is not holomorphic even if $w$ is odd. Therefore, we have the following theorem applying $[\mathrm{FHW}]$ to $D_{0}$.

Theorem 2.3 ([FHW, Theorem 4.4.3]). If $D_{0}$ is not a Hermitian symmetric domain (i.e., $L_{0} \neq K_{0}$ ), any holomorphic function on $D_{0}$ is constant.

Now the fiber of $p\left(F_{0}\right)$ is the $K_{0}$-orbit $C_{0}=K_{0} \cdot F_{0}$. We call $C_{0}$ the base cycle of $F_{0}$. By [FHW, Theorem 4.3.1], $K_{0, \mathbb{C}}$ acts on $C_{0}$ transitively, and then $C_{0}=K_{0, \mathbb{C}} \cdot F_{0}$ is a compact submanifold of $D_{0}$.

Proposition 2.4 ([FHW, Lemma 5.1.3]). Let $J=\left\{g \in G_{\mathbb{C}} \mid g C_{0}=C_{0}\right\}$. Then $J$ is a closed complex subgroup of $G_{\mathbb{C}}$. The quotient manifold $\mathcal{M}_{\check{D}}=$ $\left\{g C_{0} \mid g \in G_{\mathbb{C}}\right\} \cong G_{\mathbb{C}} / J$ has a natural structure of $G_{\mathbb{C}}$-homogeneous complex manifold, and the subset $\left\{g C_{0} \mid g \in G_{\mathbb{C}}\right.$ and $\left.g C_{0} \subset D\right\}$ is open in $\mathcal{M}_{\check{D}}$.

The topological component of $C_{0}$ in $\left\{g C_{0} \mid g \in G_{\mathbb{C}}\right.$ and $\left.g C_{0} \subset D_{0}\right\}$ is called the cycle space of $D_{0}$. We denote the cycle space of $D_{0}$ by $\mathcal{M}_{D_{0}}$. If $D_{0}$ is Hermitian symmetric, the projection $p$ is trivial; therefore, $\mathcal{M}_{D_{0}}=D_{0}$.

\subsection{Cycle spaces for odd weight cases}

We describe cycle spaces explicitly in the odd weight case when $D_{0}$ is not Hermitian symmetric according to [FHW, Section 5.5B]. In this case $D=D_{0}$. For a base point $F_{0} \in D$, we define

$$
f_{\text {even }}^{p}=\sum_{\substack{r \geq p, r: \text { even }}} h^{r, s}, \quad f_{\text {odd }}^{p}=\sum_{\substack{r \geq p, r: \text { odd }}} h^{r, s} .
$$

Let $V$ and $W$ be $\langle$,$\rangle -isotropic subspaces, and let$

$$
C_{V, W}=\left\{F \in \check{D} \mid \operatorname{dim}\left(F^{p} \cap V\right)=f_{\text {even }}^{p}, \operatorname{dim}\left(F^{p} \cap W\right)=f_{\text {odd }}^{p}\right\} .
$$


Here $g C_{V, W}=C_{g V, g W}$ for $g \in G_{\mathbb{C}}$ by the definition. Now

$$
\begin{aligned}
F_{0}^{p} \cap H_{\text {even }} & =\bigoplus_{\substack{r \geq p, r: \text { even }}} H^{r, s}, \\
g F_{0}^{p} \cap H_{\text {even }} & =g\left(F_{0}^{p} \cap H_{\text {even }}\right)=\bigoplus_{\substack{r \geq p, r: \text { even }}} g H^{r, s}
\end{aligned}
$$

for $g \in K$. Then $C_{0}=C_{H^{\text {even }}, H^{\text {odd }}}$.

By $(2.3), G_{\mathbb{R}} / K$ is isomorphic to the Siegel space $\mathcal{B}$. In this case, the cycle space $\mathcal{M}_{D}$ is described as follows.

Proposition 2.5. We have

$$
\mathcal{M}_{D}=\left\{C_{V, W} \mid V<0 \text { and } W>0 \text { for } i^{w}\langle\bullet, \bar{\bullet}\rangle\right\} \cong \mathcal{B} \times \overline{\mathcal{B}},
$$

where $w$ is the weight.

Proof. Now the $G_{\mathbb{R}^{-o r b i t}} G_{\mathbb{R}} H^{\text {even }}$ is isomorphic to $\mathcal{B}$. Then $G_{\mathbb{R}} H^{\text {odd }}$ is the complex conjugate $\overline{\mathcal{B}}$. Since $\mathcal{B}$ (resp., $\overline{\mathcal{B}}$ ) is an open subset of the flag manifold $G_{\mathbb{C}} H^{\text {even }}$ (resp., $G_{\mathbb{C}} H^{\text {odd }}$ ), we have $\mathcal{B} \times \overline{\mathcal{B}} \subset G_{\mathbb{C}} H^{\text {even }} \times G_{\mathbb{C}} H^{\text {odd }}$. For $\left(H^{\text {even }}, H^{\text {odd }}\right) \in G_{\mathbb{C}} H^{\text {even }} \times G_{\mathbb{C}} H^{\text {odd }}$, the $G_{\mathbb{C}^{-} \text {-orbit }} G_{\mathbb{C}}\left(H^{\text {even }}, H^{\text {odd }}\right)$ includes $\mathcal{B} \times \overline{\mathcal{B}}$ by [FHW, Lemma 5.4.1]. Now the isotropy subgroup of $G_{\mathbb{C}}$ at $\left(H^{\text {even }}, H^{\text {odd }}\right)$ is $K_{\mathbb{C}}$. Then we have $G_{\mathbb{C}} / K_{\mathbb{C}} \supset \mathcal{B} \times \overline{\mathcal{B}}$.

Since $\mathcal{M}_{\check{D}} \cong G_{\mathbb{C}} / J$ and $K_{\mathbb{C}} \subset J$, we have the projection

$$
\pi: G_{\mathbb{C}} / K_{\mathbb{C}} \rightarrow \mathcal{M}_{\check{D}} ; \quad g \quad\left(\bmod K_{\mathbb{C}}\right) \mapsto g C_{0}
$$

By [FHW, Proposition 5.4.3], $\pi$ is injective on $\mathcal{B} \times \overline{\mathcal{B}}$. Moreover, by [FHW, Theorem 5.5.1], $\pi(\mathcal{B} \times \overline{\mathcal{B}})=\mathcal{M}_{D} \subset \mathcal{M}_{\check{D}}$.

\section{§3. Moduli spaces of polarized log Hodge structures}

In this section, we review the construction of moduli spaces of log Hodge structures and state the fundamental properties following [KU] in Section 3.1. We state the main result in Section 3.2.

\subsection{Construction and fundamental properties}

We call $\sigma \subset \mathfrak{g}_{\mathbb{R}}$ a nilpotent cone if it satisfies the following conditions:

(1) $\sigma$ is a closed cone generated by finitely many elements of $\mathfrak{g}_{\mathbb{Q}}$;

(2) $N \in \sigma$ is nilpotent as an endomorphism of $H_{\mathbb{R}}$;

(3) $N N^{\prime}=N^{\prime} N$ for any $N, N^{\prime} \in \sigma$.

For $A=\mathbb{R}, \mathbb{C}$, we denote by $\sigma_{A}$ the $A$-linear span of $\sigma$ in $\mathfrak{g}_{A}$. 
Definition 3.1. Let $\sigma=\sum_{j=1}^{n} \mathbb{R}_{\geq 0} N_{j}$ be a nilpotent cone, and let $F \in \check{D}$. Then

$$
\exp \left(\sigma_{\mathbb{C}}\right) F \subset \check{D}
$$

is called a $\sigma$-nilpotent orbit if it satisfies the following conditions:

(1) $\exp \left(\sum_{j} i y_{j} N_{j}\right) F \in D$ for all $y_{j} \gg 0$;

(2) $N F^{p} \subset F^{p-1}$ for all $p \in \mathbb{Z}$ and for all $N \in \sigma$.

We define the set of nilpotent orbits

$$
D_{\sigma}:=\{(\tau, Z) \mid \tau: \text { face of } \sigma, Z \text { is a } \tau \text {-nilpotent orbit }\}
$$

For a nilpotent cone $\sigma$, we have the abelian group and the monoid

$$
\Gamma(\sigma)^{\mathrm{gp}}=\exp \left(\sigma_{\mathbb{R}}\right) \cap G_{\mathbb{Z}}, \quad \Gamma(\sigma)=\exp (\sigma) \cap G_{\mathbb{Z}}
$$

We define a geometric structure on $\Gamma(\sigma)^{\mathrm{gp}} \backslash D_{\sigma}$. First, we review some basic facts about toric varieties. The monoid $\Gamma(\sigma)$ defines the toric varieties

$$
\begin{aligned}
\operatorname{toric}_{\sigma} & :=\operatorname{Spec}\left(\mathbb{C}\left[\Gamma(\sigma)^{\vee}\right]\right)_{\text {an }} \cong \operatorname{Hom}\left(\Gamma(\sigma)^{\vee}, \mathbb{C}\right), \\
\operatorname{torus}_{\sigma} & :=\operatorname{Spec}\left(\mathbb{C}\left[\Gamma(\sigma)^{\vee \mathrm{gp}}\right]\right)_{\mathrm{an}} \cong \operatorname{Hom}\left(\Gamma(\sigma)^{\vee \mathrm{gp}}, \mathbb{G}_{m}\right) \cong \mathbb{G}_{m} \otimes \Gamma(\sigma)^{\mathrm{gp}},
\end{aligned}
$$

where $\mathbb{C}$ in the right-hand side of the first line is regarded as a semigroup via multiplication and the above homomorphisms are of semigroups. As in [F, Section 2.1], we choose for a face $\tau$ of $\sigma$ the distinguished point

$$
x_{\tau}: \Gamma(\sigma)^{\vee} \rightarrow \mathbb{C} ; \quad u \mapsto \begin{cases}1 & \text { if } u \in \Gamma(\tau)^{\perp}, \\ 0 & \text { otherwise. }\end{cases}
$$

Then toric $\sigma$ can be decomposed by torus orbits as

$$
\text { toric }_{\sigma}=\bigsqcup_{\tau: \text { face of } \sigma}\left(\operatorname{torus}_{\sigma} \cdot x_{\tau}\right) .
$$

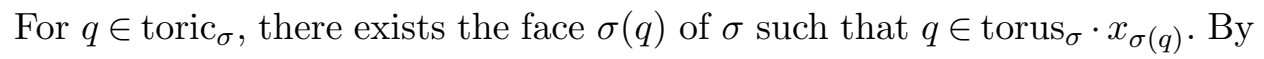
a surjective homomorphism

$$
\mathbf{e}: \sigma_{\mathbb{C}} \rightarrow \text { torus }_{\sigma} \cong \mathbb{G}_{m} \otimes \Gamma(\sigma)^{\mathrm{gp}} ; \quad w \log (\gamma) \mapsto \exp (2 \pi \sqrt{-1} w) \otimes \gamma,
$$

$q$ can be written as $q=\mathbf{e}(z) \cdot x_{\sigma(q)}$. Here $\operatorname{ker}(\mathbf{e})=\log \left(\Gamma(\sigma)^{\mathrm{gp}}\right)$, and $z$ is determined uniquely modulo $\log \left(\Gamma(\sigma)^{\mathrm{gp}}\right)+\sigma(q)_{\mathbb{C}}$. 
We define the analytic space $\check{E}_{\sigma}:=$ toric $_{\sigma} \times \check{D}$ and the subset

$$
E_{\sigma}:=\left\{\begin{array}{l|l}
(q, F) \in \check{E}_{\sigma} & \begin{array}{c}
\exp \left(\sigma(q)_{\mathbb{C}}\right) \exp (z) F \text { is a } \sigma(q) \text {-nilpotent orbit } \\
\text { where } q=\mathbf{e}(z) \cdot x_{\sigma(q)}
\end{array}
\end{array}\right\} .
$$

Here we endow $E_{\sigma}$ with the strong topology (see [KU, Section 3.1]) in $\check{E}_{\sigma}$. We then define the canonical map

$$
\begin{aligned}
\pi: E_{\sigma} & \rightarrow \Gamma(\sigma)^{\mathrm{gp}} \backslash D_{\sigma}, \\
(q, F) & \mapsto\left(\sigma(q), \exp \left(\sigma(q)_{\mathbb{C}}\right) \exp (z) F\right) \quad \bmod \Gamma(\sigma)^{\mathrm{gp}} .
\end{aligned}
$$

We endow $\Gamma(\sigma)^{\mathrm{gp}} \backslash D_{\sigma}$ with the strongest topology for which the maps $\pi$ are continuous. Kato and Usui give the geometric properties of $E_{\sigma}, \Gamma(\sigma)^{\mathrm{gp}} \backslash D_{\sigma}$ and $E_{\sigma} \rightarrow \Gamma(\sigma)^{\mathrm{gp}} \backslash D_{\sigma}$ by using the language log manifolds (see [KU, Section 3.5]).

THEOREM 3.2 ([KU, Theorem A]). We have the following:

(1) $E_{\sigma}$ and $\Gamma(\sigma)^{\mathrm{gp}} \backslash D_{\sigma}$ are logarithmic manifolds;

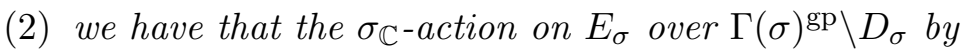

$$
a \cdot(q, F):=(\mathbf{e}(a) q, \exp (-a) F) \quad\left(a \in \sigma_{\mathbb{C}},(q, F) \in E_{\sigma}\right),
$$

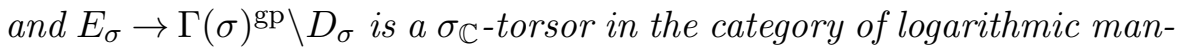
ifolds.

Log manifolds are roughly analytic spaces with slits. A typical example of a log manifold is

$$
\mathbb{C}^{2} \supset\{(x, y) \mid x=0 \Rightarrow y=0\}
$$

which is defined by the $\log$ differential 1-form $y d \log x$ of the $\log$ analytic space $\mathbb{C}^{2}$.

Moreover, Kato and Usui in [KU] define polarized log Hodge structures (see [KU, Section 2.4]), and they show that $\Gamma(\sigma)^{\mathrm{gp}} \backslash D_{\sigma}$ is a fine moduli space of polarized log Hodge structures (see [KU, Theorem B]).

In the classical situation, $\Gamma(\sigma)^{\mathrm{gp}} \backslash D_{\sigma}$ is just a toroidal partial compactification and the boundary is of codimension 1 (see $[\mathrm{N}]$ ). However, the codimension may be greater than 1 in the nonclassical situation.

EXAmple 3.3 (The $(1,1,1,1)$-case). Nilpotent orbits in the case where the Hodge numbers are $h^{3,0}=h^{0,3}=1$ and $h^{1,2}=h^{2,1}=1,0$ otherwise (we 
call it the $(1,1,1,1)$-case) are classified by [KU, Section 12.3] or [GGK1]. In this case, $D \cong \operatorname{Sp}(2, \mathbb{R}) /(U(1) \times U(1))$ and $\operatorname{dim} D=4$. Here $D$ is not a Hermitian symmetric space. All possible nilpotent cones are of rank 1 . For a nilpotent orbit $\left(\mathbb{R}_{\geq 0} N, \exp (\mathbb{C} N) F\right)$, we have the limiting mixed Hodge structure $(W(N), F)$ by $[\mathrm{S}]$ twisting $W(N)$. Here $(W(N), F)$ is one of the following types:

Type-I: $N^{2}=0, \operatorname{dim}(\operatorname{Im} N)=1 . \quad$ Type-II: $N^{2}=0, \operatorname{dim}(\operatorname{Im} N)=2$.

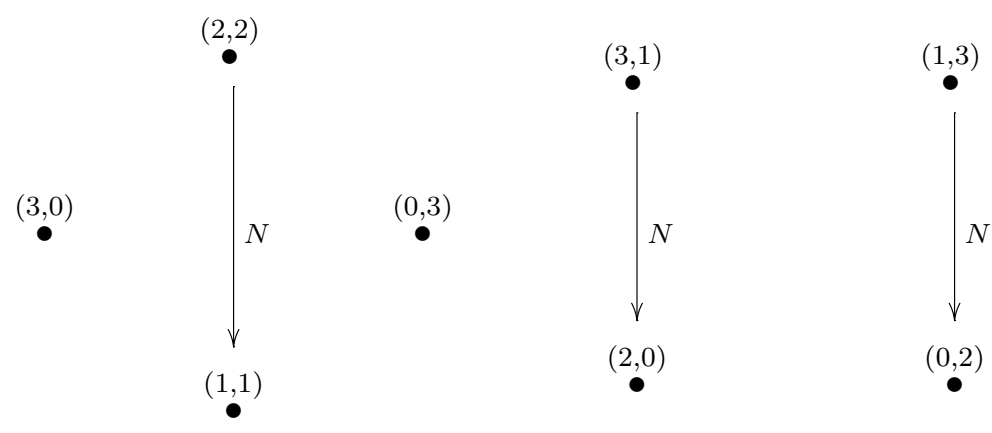

Type-III: $N^{3} \neq 0, N^{4}=0$.

Dimensions of boundaries

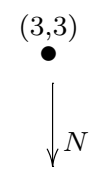

$(2,2)$

\begin{tabular}{|c|c|}
\hline & $\operatorname{dim}\left(D_{\sigma}-D\right)$ \\
\hline Type-I & 2 \\
\hline Type-II & 1 \\
\hline Type-III & 1 \\
\hline
\end{tabular}

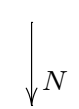

$\stackrel{(1,1)}{\bullet} N$

$(0,0)$

Geometrically, type-I or type-III degeneration occurs in the quintic-mirror family, and type-II degeneration occurs in the Borcea-Voisin mirror family (see [GGK1, Part III.A], [U2]).

\subsection{Whether the torsors are trivial}

By Theorem 3.2, we have the torsor $E_{\sigma} \rightarrow \Gamma(\sigma)^{\mathrm{gp}} \backslash D_{\sigma}$ for a period domain $D$ and a nilpotent cone $\sigma$. In [H1], we showed the triviality of torsors in the classical situation. We show a nontriviality of the torsors in the nonclassical 
situation by using the fact that any holomorphic function on $D$ is constant in the nonclassical case (see Theorem 2.3).

THEOREM 3.4. Let $D$ be a period domain (for pure Hodge structures), and let $(\sigma, Z)$ be a nilpotent orbit. Then $E_{\sigma} \rightarrow \Gamma(\sigma)^{\mathrm{gp}} \backslash D_{\sigma}$ is trivial if and only if $D$ is Hermitian symmetric or $\sigma=\{0\}$.

Proof. By [H1, Theorem 5.6], the torsors are trivial if $D$ is a Hermitian symmetric space. If $\sigma=\{0\}$, the torsor is just the identity map $D \rightarrow D$; therefore, the torsor is trivial. Thus it suffices to show that the torsor is nontrivial if $D$ is not Hermitian symmetric.

We assume that $\pi: E_{\sigma} \rightarrow \Gamma(\sigma)^{\text {gp }} \backslash D_{\sigma}$ is trivial for a non-Hermitian symmetric space $D$ and for a nilpotent cone $\sigma \neq\{0\}$. Now

$$
\pi^{-1}\left(\Gamma(\sigma)^{\mathrm{gp}} \backslash D\right)=E_{\sigma} \cap\left(\operatorname{torus}_{\sigma} \times \check{D}\right)
$$

by the definition of $E_{\sigma}$, and this is a complex analytic space since torus ${ }_{\sigma} \times \check{D}$ has trivial log structure. Thus the restriction of the torsor to $\pi^{-1}\left(\Gamma(\sigma)^{\operatorname{gp}} \backslash D\right)$ is a torsor in the category of complex analytic spaces, and we have a section $\Gamma(\sigma)^{\mathrm{gp}} \backslash D_{\sigma} \rightarrow E_{\sigma}$ and a holomorphic map $\Phi: D \rightarrow\left(\mathbb{C}^{*}\right)^{l}$ such that we have the following diagram:

$$
\Gamma(\sigma)^{\mathrm{gp}} \backslash D_{\sigma} \longrightarrow E_{\sigma}
$$

$$
\begin{gathered}
\cup \\
\cup: D \stackrel{\text { quot. }}{\longrightarrow} \Gamma(\sigma)^{\mathrm{gp}} \backslash D \longrightarrow E_{\sigma} \cap\left(\operatorname{torus}_{\sigma} \times \check{D}\right) \stackrel{\text { proj. }}{\longrightarrow} \text { torus }_{\sigma} \cong\left(\mathbb{C}^{*}\right)^{l},
\end{gathered}
$$

where $l=\operatorname{rank} \Gamma(\sigma)^{\mathrm{gp}}$.

For a nilpotent $N$ in the relative interior of $\sigma$, we have

$$
\lim _{y \rightarrow \infty} \exp (i y N) F=\left(\sigma, \exp \left(\sigma_{\mathbb{C}}\right) F\right)
$$

through $D \rightarrow \Gamma(\sigma)^{\mathrm{gp}} \backslash D \hookrightarrow \Gamma(\sigma)^{\mathrm{gp}} \backslash D_{\sigma}$ by [KU, Proposition 3.4.4]. Then $\Phi(\exp (i y N) F)$ has to converge to $0 \in$ toric $_{\sigma}$ as $y \rightarrow \infty$. This contradicts Theorem 2.3.

\section{$\S 4$. Remarks on [H1]}

We showed the nontriviality of the torsor in [H1, Proposition 5.8] using a method different from that used in Theorem 3.4. We formulate it by using 
the SL(2)-orbit theorem and cycle spaces, and we give a second proof of the nontriviality result for a special case. While this second proof requires some special conditions, the result is stronger than the first one since it says that there exists no section over certain open sets around a boundary point. A property of some cycle spaces induces this result. We observe the property of cycle spaces in the $(1,1,1,1)$-case explicitly in Section 4.3 . In this section, we assume that $D$ is not Hermitian symmetric.

\section{1. $\mathrm{SL}(2)$-orbits and cycle spaces}

Let $\left(\mathbb{R}_{\geq 0} N, \exp (\mathbb{C} N) F\right)$ be a nilpotent orbit. By $[\mathrm{S}]$ there exists the monodromy weight filtration $W(N)$, and $(W(N), F)$ is a mixed Hodge structure. By [CKS, Proposition 2.20] there exists the $\mathbb{R}$-split mixed Hodge structure $(W(N), \hat{F})$ associated to it. We then have the Deligne decomposition $H_{\mathbb{C}}=\bigoplus_{p, q} I^{p, q}$ for $(W(N), \hat{F})$, where

$$
\hat{F}^{p}=\bigoplus_{r \leq p} I^{r, s}, \quad W(N)_{k}=\bigoplus_{r+s=k} I^{r, s}, \quad \overline{I^{p, q}}=I^{q, p} .
$$

By the SL(2)-orbit theorem (see [S, Theorem 5.13], [CKS, Section 3]), there exists the Lie group homomorphism $\rho: \operatorname{SL}(2, \mathbb{C}) \rightarrow G_{\mathbb{C}}$ defined over $\mathbb{R}$ and the holomorphic map $\phi: \mathbb{P}^{1} \rightarrow \check{D}$ satisfying the following conditions:

(S1) $\rho(g) \phi(z)=\phi(g z)$;

(S2) $\phi(0)=\hat{F}$

(S3) $\rho_{*}\left(\mathbf{n}_{-}\right)=N$;

(S4) $H v=(p+q-w) v$ for $v \in I^{p, q}$, where $\rho_{*}(\mathbf{h})=H$;

$(\mathrm{S} 5) \rho_{*}: \mathfrak{s l}(2, \mathbb{C}) \rightarrow \mathfrak{g}_{\mathbb{C}}$ is a $(0,0)$-morphism of Hodge structure, where $\mathfrak{g}_{\mathbb{R}}$ (resp., $\mathfrak{s l}(2, \mathbb{R}))$ has a Hodge structure of weight 0 relative to $\phi(i)$ (resp., $i)$,

where $\left\{\mathbf{n}_{-}, \mathbf{h}, \mathbf{n}_{+},\right\}$is the $s l_{2}$-triple (see Example 2.1 ).

Let $F_{0}=\phi(i)$ be a base point of $D$. We write

$$
\rho_{*}\left(\mathbf{n}_{+}\right)=N^{+}, \quad X=\frac{1}{2}\left(i N-H+i N^{+}\right) .
$$

Then $X \in \mathfrak{g}_{0}^{-1,1}$ by (S5) and (2.1), where $\mathfrak{g}_{0}^{-1,1}$ is the $(-1,1)$-component of the Hodge decomposition of $\mathfrak{g}_{\mathbb{C}}$ with respect to $F_{0}$. By $(\mathrm{S} 1)$ we have

$$
\exp (z X) \phi(i)=\phi\left(\frac{1+z}{1-z} i\right)
$$


therefore,

$$
\exp \left(\frac{y}{2+y} X\right) \phi(i)=\phi((1+y) i)=\exp (i y N) \phi(i)
$$

Lemma 4.1. Let $C_{0}$ be the base cycle of $F_{0}$. If $\operatorname{dim}(\operatorname{Im} N)=1$, then both (A) and (B) hold:

(A) there exists $F_{\text {fix }} \in C_{0}$ such that $\exp (X) F_{\text {fix }}=F_{\text {fix }}$;

(B) $\exp (z X) C_{0} \subset D$ (i.e., $\left.\exp (z X) C_{0} \in \mathcal{M}_{D}\right)$ for $|z|<1$.

Proof. At first we write $X$ explicitly. Considering the type of the limiting Hodge structure $(W(N), F)$, the case where $\operatorname{dim}(\operatorname{Im} N)=1$ is possible only if the weight is $2 m-1$ and $\operatorname{dim}\left(\mathrm{Gr}_{2 m}^{W}\right)=\operatorname{dim}\left(\mathrm{Gr}_{2 m-2}^{W}\right)=1$. We then have an $\mathbb{R}$-element $e$ in the $(m, m)$-component $I^{m, m}$ of the Deligne decomposition of $(W(N), \hat{F})$. Here $X$ is given by

$$
\begin{aligned}
e & \mapsto \frac{1}{2}(-e+i N e), \quad N e \mapsto \frac{1}{2}(i e+N e)=-i X e, \\
I^{p, q} & \rightarrow 0 \quad \text { for } p+q=2 m-1 .
\end{aligned}
$$

We write $u=\exp (i N) e$. Since $e \in \hat{F}^{m}, u \in \exp (i N) \hat{F}^{m}=F_{0}^{m}$. Moreover, since $N e \in \hat{F}^{m-1}$,

$$
N e=\exp (i N) N e \in \exp (i N) \hat{F}^{m-1}=F_{0}^{m-1} .
$$

Then

$$
\bar{u}=e-i N e=u-2 i N e \in F_{0}^{m-1} .
$$

Hence $u$ is in the $(m, m-1)$-component $H_{0}^{m, m-1}$ of the Hodge decomposition for $F_{0}$. Here

$$
X u=-e+i N e=-\bar{u}, \quad X w=0 \quad \text { if }\langle w, \bar{u}\rangle=0 .
$$

First we show (A). We denote by $\|\bullet\|$ the norm induced by the positive definite Hermitian form $\left\langle C_{F_{0}} \bullet, \bar{\bullet}\right\rangle$, where $C_{F_{0}}$ is the Weil operator for $F_{0}$. Scaling $u$, we may assume that $\|u\|=1$. We take $v \in H_{0}^{m-2, m+1}$ such that $\|v\|=1$. We define $g \in \operatorname{Aut}\left(H_{\mathbb{C}}\right)$ by

$$
g u=v, \quad g v=u, \quad g \bar{v}=\bar{u}, \quad g \bar{u}=\bar{v},
$$

and $g w=w$ if $w$ is vertical to $u, v, \bar{u}$, and $\bar{v}$ for $\langle$,$\rangle . Then$

$$
g u=v=\overline{g \bar{u}}=\bar{g} u, \quad g v=u=\bar{g} \overline{\bar{v}}=\bar{g} v .
$$

Therefore, $g$ is defined over $\mathbb{R}$ and preserves the polarization $\langle$,$\rangle ; that is,$ $g \in G_{\mathbb{R}}$. Moreover, $g \in K$ since $g$ preserves $H^{\text {even }}$. 
Claim. We have that $g F_{0} \in C_{0}$ is a fixed point for $\exp (X)$.

Proof. Now $u=g v \in g H_{0}^{m-2, m+1}$. By (4.3), it suffices to show that $X u \in$ $g F_{0}^{m-2}$. In fact,

$$
X u=-\bar{u}=-g \bar{v} \in g H_{0}^{m+1, m-2} .
$$

Next we show (B). We take a unitary basis $\left\{u_{1}, \ldots, u_{l}\right\}$ of $H_{0}^{m, m-1}$. We may assume that $u_{1}=u$. Then $\exp (X) u_{j}=u_{j}$ if $j \neq 1$ and $\exp (z X) u_{1}=$ $u_{1}-z \bar{u}_{1}$. Here

$$
i\left\langle\exp (z X) u_{1}, \overline{\exp (z X) u_{1}}\right\rangle=\left\|u_{1}\right\|^{2}-|z|^{2}\left\|u_{1}\right\|^{2}=1-|z|^{2} .
$$

By Proposition 2.5, $\exp (z X) C_{0} \subset D$ if and only if $|z|<1$.

REMARK 4.2. In the $(1,1,1,1)$-case, a type-I nilpotent $N$ satisfies $\operatorname{dim}(\operatorname{Im} N)=1$, however other types do not. Above, (4.2) and items (A) and (B) of Lemma 4.1 correspond to the conditions (5.4), (5.6), and (5.5) of [H1, Section 5], respectively.

\subsection{Nontriviality on some open sets}

Let $\left(\mathbb{R}_{\geq 0} N, \exp (\mathbb{C} N) F\right)$ be a nilpotent orbit. Let $(\rho, \phi)$ be the $\operatorname{SL}(2)$-orbit associated to $(N, F)$. Taking $F_{0}=\phi(i)$ as a base point, we have the base cycle $C_{0}$ and $X \in \mathfrak{g}_{0}^{-1,1}$ as in (4.1). We define the subset

$$
\mathcal{M}(\varepsilon)=\left\{\exp (\alpha X) C_{0} \mid 1-\varepsilon<\alpha<1\right\} \subset \mathcal{M}_{\check{D}}
$$

for $0<\varepsilon$. If $\operatorname{dim}(\operatorname{Im} N)=1$, by Lemma 4.1 (B),

$$
\exp (\alpha X) C_{0} \in \mathcal{M}_{D} \quad \text { for }-1<\alpha<1, \exp (X) C_{0} \notin \mathcal{M}_{D} .
$$

Then $\mathcal{M}(\varepsilon)$ is a nearby set of the boundary point $\exp (X) C_{0} \in \overline{\mathcal{M}}_{D}$.

Proposition 4.3. Let $U$ be an open set including the boundary point $\left(\sigma, \exp \left(\sigma_{\mathbb{C}}\right) \hat{F}\right)$ in $\Gamma(\sigma)^{\mathrm{gp}} \backslash D_{\sigma}$, where $\sigma=\mathbb{R}_{\geq 0} N$ with $\operatorname{dim}(\operatorname{Im} N)=1$. If there exists $0<\varepsilon<1$ such that $q(C) \subset U$ for any $C \in \mathcal{M}(\varepsilon)$ and the quotient map $q: D \rightarrow \Gamma(\sigma)^{\mathrm{gp}} \backslash D$, then no section over the open set $U$ exists.

Proof. We assume that there exists a local trivialization over $U$. Similar to the proof of Theorem 3.4, we have a section $U \rightarrow E_{\sigma}$ and the holomorphic map $\Phi: q^{-1}(U) \rightarrow \mathbb{C}^{*}$ given by the following diagram

$$
\begin{aligned}
& U \longrightarrow E_{\sigma} \\
& \cup \quad \cup \\
& \Phi: q^{-1}(U) \longrightarrow U \cap\left(\Gamma(\sigma)^{\mathrm{gp}} \backslash D\right) \longrightarrow E_{\sigma} \cap\left(\mathbb{C}^{*} \times \check{D}\right) \longrightarrow \mathbb{C}^{*} .
\end{aligned}
$$


By (4.2) and the assumption, we have

$$
q\left(\exp \left(\frac{y}{2+y} X\right) F_{0}\right)=q\left(\exp (i y N) F_{0}\right) \subset U
$$

for

$$
1-\varepsilon<\frac{y}{2+y}<1, \quad \text { that is, } \frac{2(1-\varepsilon)}{\varepsilon} \leq y .
$$

By $(3.2), \Phi\left(\exp (i y N) F_{0}\right)$ has to converge to $0 \in$ toric $_{\sigma}$ as $y \rightarrow \infty$.

Now $\Phi$ is constant on the compact complex submanifold $C \in \mathcal{M}(\varepsilon)$. By Lemma 4.1, we then have

$$
\begin{aligned}
\Phi\left(\exp (i y N) F_{0}\right) & =\Phi\left(\exp \left(\frac{y}{2+y} X\right) F_{0}\right)=\Phi\left(\exp \left(\frac{y}{2+y} X\right) F_{\text {fix }}\right) \\
& =\Phi\left(\exp \left(\frac{y^{\prime}}{2+y^{\prime}} X\right) F_{\text {fix }}\right)=\Phi\left(\exp \left(i y^{\prime} N\right) F_{0}\right)
\end{aligned}
$$

for $y, y^{\prime}>2(1-\varepsilon) / \varepsilon$. This contradicts the convergence of $\Phi\left(\exp (i y N) F_{0}\right)$.

Remark 4.4. Above $X, F_{\text {fix }}$ and $F_{0}$ correspond to the notations $N^{\prime}, F_{\infty}$ and $F_{0}$ in [H1, Section 5], respectively.

\subsection{The $(1,1,1,1)$-case}

The conditions (A) and (B) of Lemma 4.1 induce Proposition 4.3. In our later work [H2], condition (B) also plays an important role in the study of boundary structure. We then expect that $\Gamma \backslash D_{\Sigma}$ has good properties if $\Sigma$ satisfies the conditions (A) or (B). Therefore, it is important to determine which cone satisfies (A) or (B). As we saw in Example 3.3, the types of nilpotent orbits in the $(1,1,1,1)$-case are well known. Type-I nilpotents satisfy $(\mathrm{A})$ and $(\mathrm{B})$. We show that $(\mathrm{A})$ or $(\mathrm{B})$ does not hold in other types below.

Let $\left(\mathbb{R}_{\geq 0} N, \exp (\mathbb{C} N) F\right)$ be a nilpotent orbit, and let $(\rho, \phi)$ be the $\operatorname{SL}(2)$ orbit associated $(N, F)$. We can choose a unitary basis

$$
u_{3} \in H_{0}^{3,0}, \quad u_{2} \in H_{0}^{2,1}, \quad \bar{u}_{2} \in H_{0}^{1,2}, \quad \bar{u}_{3} \in H_{0}^{0,3}
$$

for the Hodge decomposition for $F_{0}=\phi(i)$. Here the base cycle of $F_{0}$ is $C_{0} \cong U(2) /(U(1) \times U(1)) \cong \mathbb{P}^{1}$. The isomorphism $\mathbb{P}^{1} \stackrel{\sim}{\rightarrow} C_{0} \subset D$ is given by

$$
\begin{aligned}
& F_{z}^{3}=\operatorname{span}_{\mathbb{C}}\left\{z \bar{u}_{2}+u_{3}\right\}, \quad F_{z}^{2}=\operatorname{span}_{\mathbb{C}}\left\{z \bar{u}_{2}+u_{3}, u_{2}-z \bar{u}_{3}\right\}, \\
& F_{\infty}^{3}=\operatorname{span}_{\mathbb{C}}\left\{\bar{u}_{2}\right\}, \quad F_{\infty}^{2}=\operatorname{span}_{\mathbb{C}}\left\{\bar{u}_{2}, \bar{u}_{3}\right\} .
\end{aligned}
$$


The properties $(\mathrm{A})$ and $(\mathrm{B})$ of Lemma 4.1 depend on $X \in \mathfrak{g}_{0}^{-1,1}$. For a type-I nilpotent, $X$ is given by
$(3,0)$

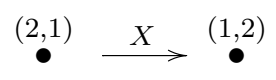
$\stackrel{(0,3)}{\bullet}\left(u_{2} \mapsto-\bar{u}_{2} \mapsto 0\right)$

We determine the type of $X$ in the case for type-II and for type-III, and consider whether $(\mathrm{A})$ or $(\mathrm{B})$ holds or not.

4.3.1. Type-II. We have the following.

Proposition 4.5. If $N$ is of type-II, then (B) holds; however, (A) does not hold.

Proof. Let $v$ be a nonzero element in $I^{3,1}$ of the Deligne decomposition of $(W(N), \hat{F})$. Then

$$
N v \in I^{2,0}, \quad \bar{v} \in I^{1,3}, \quad N \bar{v} \in I^{0,2} .
$$

We write $u_{3}=\exp (i N) v$. Since $v \in \hat{F}^{3}, u_{3} \in F_{0}^{3}=H_{0}^{3,0}$. Here the $s l_{2}$-triple is given by

$$
\begin{aligned}
N^{+} N v & =v, \quad N^{+} N \bar{v}=\bar{v}, \quad N^{+} v=N^{+} \bar{v}=0, \\
H v & =v, \quad H \bar{v}=\bar{v}, \quad H N v=-N v, \quad H N \bar{v}=-N \bar{v} .
\end{aligned}
$$

Then we have

$$
H_{0}^{2,1} \ni X u_{3}=-v+i N v=-\exp (-i N) v
$$

We write $u_{2}=X u_{3}$. Then $X u_{2}=0$. Moreover, $\bar{u}_{2} \in H_{0}^{1,2}$ and

$$
X \bar{u}_{2}=\bar{v}-i N \bar{v}=\bar{u}_{3}
$$

Summarizing these, $X \in \mathfrak{g}_{0}^{-1,1}$ is given by

$$
\stackrel{(3,0)}{\bullet} \stackrel{X}{\longrightarrow} \stackrel{(2,1) \quad(1,2)}{\bullet} \stackrel{X}{\longrightarrow} \stackrel{(0,3)}{\bullet}\left(u_{3} \mapsto u_{2} \mapsto 0, \bar{u}_{2} \mapsto \bar{u}_{3} \mapsto 0\right) .
$$

Since $X\left(z \bar{u}_{2}+u_{3}\right)=z \bar{u}_{3}+u_{2}, X F_{z}^{3} \not \subset F_{z}^{3}$ for $z \in \mathbb{P}^{1}$ in (4.4). Then there is no fixed point for $\exp (X)$ in $C_{0}$.

Next we show that (B) holds. Scaling $v$, we may assume that $\left\|u_{3}\right\|=1$. 
Claim. We have $\left\|u_{2}\right\|=1$.

Proof. Let $a=\langle v, \bar{v}\rangle, b=\langle N v, \bar{v}\rangle, c=\langle v, N \bar{v}\rangle$, and $d=\langle N v, N \bar{v}\rangle$. Then by orthogonality, we have

$$
\begin{aligned}
& \left\langle u_{3}, \bar{u}_{3}\right\rangle=a+i b-i c+d=i, \quad\left\langle u_{3}, \bar{u}_{2}\right\rangle=-a-i b-i c+d=0, \\
& \left\langle u_{2}, \bar{u}_{3}\right\rangle=-a+i b+i c+d=0 .
\end{aligned}
$$

Since $v \in \hat{F}^{3}$ and $\bar{v} \in \hat{F}^{1}, a=0$. Therefore, the simultaneous equation induces $d=0, b-c=1$, and $\left\langle u_{2}, \bar{u}_{2}\right\rangle=a-i b+i c+d=-i$.

Here $\left\{u_{3}, u_{2}, \bar{u}_{3}, \bar{u}_{2}\right\}$ is a unitary basis. Since

$$
\begin{aligned}
& -i\left\langle\exp (z X) u_{3}, \overline{\exp (z X) u_{3}}\right\rangle=\left\|u_{3}\right\|^{2}-|z|^{2}\left\|u_{2}\right\|^{2}=1-|z|^{2}, \\
& -i\left\langle\exp (z X) \bar{u}_{2}, \overline{\exp (z X) \bar{u}_{2}}\right\rangle=\left\|u_{2}\right\|^{2}-|z|^{2}\left\|u_{3}\right\|^{2}=1-|z|^{2},
\end{aligned}
$$

$\exp (z X) C_{0} \subset D$ if and only if $|z|<1$ by Proposition 2.5.

4.3.2. Type-III. We give an example of type-III which satisfies neither (A) nor (B). All nilpotent orbits of type-III are described in [GGK1] explicitly. We consider the case where $a, b=1$ and $e, f, \pi=0$ in the notation of [GGK1, (I.C.2), (I.C.10)]. Let $H_{\mathbb{Z}}=\sum_{j=0}^{3} \mathbb{Z} e_{j}$. We write

$$
e_{3}=\left(\begin{array}{l}
1 \\
0 \\
0 \\
0
\end{array}\right), \quad e_{2}=\left(\begin{array}{l}
0 \\
1 \\
0 \\
0
\end{array}\right), \quad e_{1}=\left(\begin{array}{l}
0 \\
0 \\
1 \\
0
\end{array}\right), \quad e_{0}=\left(\begin{array}{l}
0 \\
0 \\
0 \\
1
\end{array}\right)
$$

where the bilinear form is given by

$$
\left(\begin{array}{cccc}
0 & 0 & 0 & -1 \\
0 & 0 & -1 & 0 \\
0 & 1 & 0 & 0 \\
1 & 0 & 0 & 0
\end{array}\right)
$$

Let

$$
N=\left(\begin{array}{cccc}
0 & 0 & 0 & 0 \\
1 & 0 & 0 & 0 \\
0 & 1 & 0 & 0 \\
0 & 0 & -1 & 0
\end{array}\right), \quad \hat{F}^{p}=\left\{e_{3}, \ldots, e_{p}\right\} \quad(3 \geq p \geq 0)
$$


Then $N$ and $\hat{F}$ give a nilpotent orbit of type-III, where the limit mixed Hodge structure $(W(N), \hat{F})$ is $\mathbb{R}$-split.

The $s l_{2}$-triple of the SL(2)-orbit associated to this nilpotent orbit is given by

$$
H=\left(\begin{array}{cccc}
3 & 0 & 0 & 0 \\
0 & 1 & 0 & 0 \\
0 & 0 & -1 & 0 \\
0 & 0 & 0 & -3
\end{array}\right), \quad N^{+}=\left(\begin{array}{cccc}
0 & 3 & 0 & 0 \\
0 & 0 & 4 & 0 \\
0 & 0 & 0 & -3 \\
0 & 0 & 0 & 0
\end{array}\right)
$$

Then

$$
X=\frac{1}{2}\left(\begin{array}{cccc}
-3 & 3 i & 0 & 0 \\
i & -1 & 4 i & 0 \\
0 & i & 1 & -3 i \\
0 & 0 & -i & 3
\end{array}\right)
$$

Proposition 4.6. For the above example, both (A) and (B) do not hold. Proof. Let

$$
u_{3}=\frac{\sqrt{3}}{2} \exp (i N) e_{3}=\frac{\sqrt{3}}{12}\left(\begin{array}{c}
6 \\
6 i \\
-3 \\
i
\end{array}\right)
$$

Then $\left\|u_{3}\right\|=1$. Now

$$
\begin{aligned}
& X u_{3}=\frac{\sqrt{3}}{4}\left(\begin{array}{c}
-6 \\
-2 i \\
-1 \\
i
\end{array}\right), \quad X^{2} u_{3}=\frac{\sqrt{3}}{2}\left(\begin{array}{c}
6 \\
-2 i \\
1 \\
i
\end{array}\right)=-2 \overline{X u_{3}} \\
& X^{3} u_{3}=\frac{\sqrt{3}}{2}\left(\begin{array}{c}
-6 \\
6 i \\
3 \\
i
\end{array}\right)=-6 \bar{u}_{3} .
\end{aligned}
$$

Here $\left\|X u_{3}\right\|=3$. Letting $u_{2}=X u_{3} / \sqrt{3}$, we then have a unitary basis $\left\{u_{3}, u_{2}\right.$, $\left.\bar{u}_{3}, \bar{u}_{2}\right\}$. Endomorphism $X$ gives the map

$$
\begin{gathered}
\stackrel{(3,0)}{\bullet} \stackrel{x}{\longrightarrow} \stackrel{(2,1)}{\bullet} \stackrel{x}{\longrightarrow} \stackrel{(1,2)}{\bullet} \stackrel{x}{\longrightarrow} \stackrel{(0,3)}{\bullet} \\
\left(u_{3} \mapsto \sqrt{3} u_{2} \mapsto-2 \sqrt{3} \overline{u_{2}} \mapsto-6 \overline{u_{3}} \mapsto 0\right) .
\end{gathered}
$$


Then $X F_{z}^{3} \not \subset F_{z}^{3}$ for $z \in \mathbb{P}^{1}$ in (4.4), and so there is no fixed point in $C_{0}$. Moreover, for $\bar{u}_{2} \in F_{\infty}^{3}$,

$$
-i\left\langle\exp (z X) \bar{u}_{2}, \overline{\exp (z X) \bar{u}_{2}}\right\rangle=\left\|u_{2}\right\|^{2}-3|z|^{2}\left\|u_{3}\right\|^{2}=1-3|z|^{2} .
$$

Then $\exp (z X) C_{0} \not \subset D$ for $|z| \geq 1 / \sqrt{3}$.

Acknowledgments. This work was done during a visit of the author to the Université du Québec à Montréal in August 2011 and to the Johns Hopkins University as part of the Japan-U.S. Mathematics Institute (JAMI) in September 2011, and the author thanks those institutions for their hospitality and support. The author also thanks Patrick Brosnan, Steven Lu, Gregory Pearlstein, and Steven Zucker for valuable advice and warm encouragement.

\section{REFERENCES}

[AMRT] A. Ash, D. Mumford, M. Rapoport, and Y.-S. Tai, Smooth Compactification of Locally Symmetric Varieties, Math. Sci. Press, Brookline, Massachusetts, 1975. MR 0457437.

[CMP] J. Carlson, S. Müller-Stach, and C. Peters, Period Mappings and Period Domains, Cambridge Stud. Adv. Math. 85, Cambridge University Press, Cambridge, 2003. MR 2012297.

[CKS] E. Cattani, A. Kaplan, and W. Schmid, Degeneration of Hodge structures, Ann. of Math. (2) 123 (1986), 457-535. MR 0840721. DOI 10.2307/1971333.

[FHW] G. Fels, A. Huckleberry, and J. A. Wolf, Cycle Spaces of Flag Domains: A Complex Geometric Viewpoint, Progr. Math. 245, Birkhäuser, Boston, 2006. MR 2188135.

[F] W. Fulton, Introduction to Toric Varieties, Ann. of Math. Stud. 131, Princeton University Press, Princeton, 1993. MR 1234037.

[GGK1] M. Green, P. Griffiths, and M. Kerr, "Néron models and boundary components for degenerations of Hodge structures of mirror quintic type" in Curves and Abelian Varieties, Contemp. Math. 465, Amer. Math. Soc., 2008, 71-145. MR 2457736. DOI 10.1090/conm/465/09101.

[GGK2] - Mumford-Tate Groups and Domains: Their Geometry and Arithmetic, Ann. of Math. Stud. 183, Princeton University Press, Princeton, 2012. MR 2918237.

[G] P. Griffiths, Periods of integrals on algebraic manifolds, I: Construction and properties of the modular varieties, Amer. J. Math. 90 (1968), 568-626. MR 0229641.

[H1] T. Hayama, On the boundary of the moduli spaces of log Hodge structures: Triviality of the torsor, Nagoya Math. J. 198 (2010), 173-190. MR 2666580.

[H2] - Boundaries of cycle spaces and degenerating Hodge structures, to appear in Asian J. Math, preprint, arXiv:1203.6770v3 [math.AG].

[KU] K. Kato and S. Usui, Classifying Spaces of Degenerating Polarized Hodge Structures, Ann. of Math. Stud. 169, Princeton University Press, Princeton, 2009. MR 2465224. 
[KP] M. Kerr and G. Pearlstein, Boundary components of Mumford-Tate domains, preprint, arXiv: 1210.5301v1 [math.AG].

[LS] Z. Lu and X. Sun, Weil-Petersson geometry on moduli space of polarized CalabiYau manifolds, J. Inst. Math. Jussieu 3 (2004), 185-229. MR 2055709. DOI 10. 1017/S1474748004000076.

[N] Y. Namikawa, Toroidal Compactification of Siegel Spaces, Lecture Notes in Math. 812, Springer, Berlin, 1980. MR 0584625.

[S] W. Schmid, Variation of Hodge structure: The singularities of the period mapping, Invent. Math. 22 (1973), 211-319. MR 0382272.

[U1] S. Usui, Complex structures on partial compactifications of arithmetic quotients of classifying spaces of Hodge structures, Tohoku Math. J. (2) 47 (1995), 405429. MR 1344910. DOI 10.2748/tmj/1178225524.

[U2] , Generic Torelli theorem for quintic-mirror family, Proc. Japan Acad. Ser. A Math. Sci. 84 (2008), 143-146. MR 2457802.

Department of Mathematics

National Taiwan University

Taipei 106

Taiwan

Current:

Mathematical Sciences Center

Tsinghua University

Haidian District

Beijing 100084

China

tatsuki@math.tsinghua.edu.cn 